\title{
Enantioselective total synthesis of epoxyquinone natural products (-)-phyllostine, (+)-epoxydon, (+)-epiepoxydon and $(-)$-panepophenanthrin: access to versatile chiral building blocks through enzymatic kinetic resolution
}

\author{
Goverdhan Mehta* and Kabirul Islam \\ Department of Organic Chemistry, Indian Institute of Science, Bangalore 560 012, India
}

\begin{abstract}
A new enzyme mediated protocol to access versatile chiral building blocks for the synthesis of epoxyquinone natural products is delineated. Total syntheses of $(-)$-phyllostine, $(+)$-epoxydon, $(+)$-epiepoxydon and $(-)$-panepophenanthrin have been accomplished to demonstrate the efficacy of this approach.
\end{abstract}

A range of polyketide derived natural products, embodying a compact epoxyquinone derived motif $\mathbf{1}$, as the core structure, have been encountered among diverse sources like bacteria, fungi, higher plants and mollusks. ${ }^{1}$ Representative examples of such polyoxygenated cyclohexanoids are $(+)$-epoformin $\mathbf{2 a},{ }^{1 \text { a }}(+)$-epiepoformin $\mathbf{2 b},{ }^{1 \mathrm{~b}}(-)$-theobroxide $3,{ }^{\text {lc }}(-)$-phyllostine $4,{ }^{1 \mathrm{~d}}(+)$-epoxydon $\mathbf{5 a},{ }^{\text {le }}(+)$-epiepoxydon $\mathbf{5} \mathbf{b}^{1 \mathrm{f}}$ and (+)-harveynone $\mathbf{6}^{1 \mathrm{~g}}$ These and related natural products have stimulated much synthetic activity due to their structural and stereochemical diversity and their wide ranging biological activity, from phytotoxicity, anti-fungal, antibacterial and anti-tumour to various kind of enzyme inhibition. $^{2}$

More recently, a complex natural product (+)-panepophenanthrin $\mathbf{8},{ }^{3}$ derived through a biosynthetic DielsAlder reaction from a monomeric epoxyquinone precursor 7, has been isolated from the fermentation broth of the mushroom strain Panus rudis Fr. IFO8994 and has aroused considerable current interest among synthetic chemists due to its unique activity in inhibiting the ubiquitin activating enzyme (E1), which is indispensable to the ubiquitin-proteosome pathway (UPP). ${ }^{4}$

As a part of our ongoing interest in the synthesis of epoxyquinone natural products, ${ }^{4 \mathrm{c}, 5}$ we further highlight

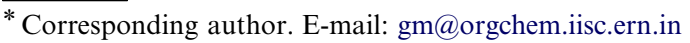<smiles>[R]C1C(C)CC(=O)C2OC21</smiles><smiles>[R]OC1C=C(C)C(=O)C2OC12</smiles><smiles>CC1=C[C@H](O)C2OC2[C@H]1O</smiles>

1. $\mathrm{R}=\mathrm{OH},=\mathrm{O}$

2a. $\mathrm{R}=\beta-\mathrm{OH},(+)$-Epoformin

3. (-)-Theobroxide 2b. $\mathrm{R}=\alpha-\mathrm{OH},(+)$-Epiepoformin<smiles>O=C1C=C(CO)C(=O)C2OC12</smiles><smiles>[R20][C@H]1C=C(CO)C(=O)[C@@H]2O[C@H]12</smiles><smiles>CC(C)C#CC1=C[C@@H](O)C2OC2C1=O</smiles>

4. (-)-Phyllostine

5a. $\mathrm{R}=\beta-\mathrm{OH},(+)$-Epoxydon

6. (+)-Harveynone 5b. $\mathrm{R}=\alpha-\mathrm{OH},(+)$-Epiepoxydon

here the efficacy of the readily available Diels-Alder adduct $9^{6}$ of cyclopentadiene and $p$-benzoquinone and its epoxide 10 as versatile building blocks for the synthesis of natural products embodying the structural motif 1 . A notable feature of the efforts outlined here is the convenient and efficient enzyme mediated kinetic resolution of a derivative of $\mathbf{1 0}$ to provide access to both the enantiomeric forms of the core structure 1. One of these 


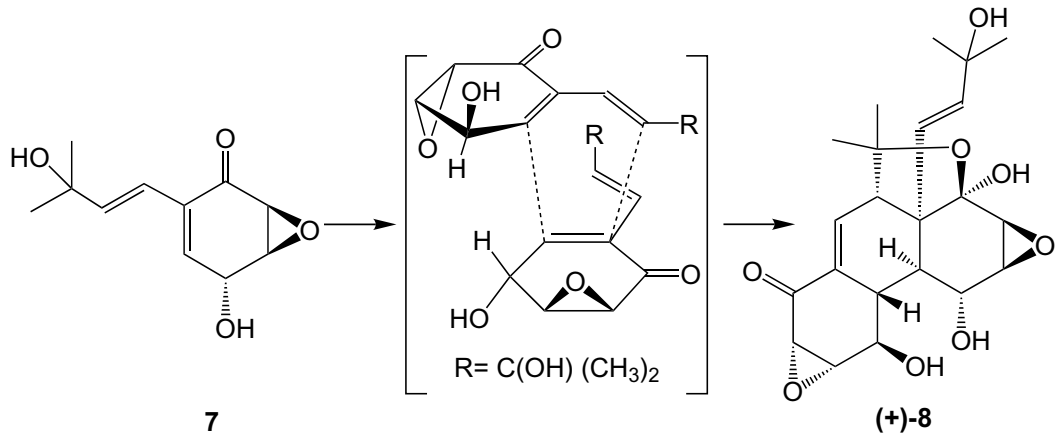

enantiomers has been elaborated to the natural products $(-)$-phyllostine 4, (+)-epoxydon 5a and (+)-epiepoxydon $\mathbf{5 b}$ and also utilized for the total synthesis of $(-)$ panapophenanthrin $\mathbf{8}$, the antipode of the biologically important natural product $(+)-8$. These endeavours towards the total synthesis of epoxyquinone natural products constitute the theme of this letter.

Readily available tricyclic endo-adduct 9 can be conveniently transformed to $\mathbf{1 0}^{\mathbf{7}}$ in high yield and further exposure to formalin solution in the presence of catalytic amounts of DBU under controlled conditions led stereoselectively to the $\alpha$-hydroxymethylated product $\mathbf{1 1}$ in excellent yield (Scheme 1). TBS-protection of the hydroxyl group in $\mathbf{1 1}$ to yield $\mathbf{1 2}$ and sodium borohydride reduction stereoselectively furnished the endo-alcohol 13 (Scheme 1). ${ }^{7}$ After some trial experimentation, it was found that $\mathbf{1 3}$ was amenable to efficient enzymatic kinetic resolution through transesterification. ${ }^{8}$ Thus, exposure of $( \pm)$-13 to lipase PS-D in vinyl acetate solvent and termination of the reaction at nearly $50 \%$ transesterification led to the isolation of hydroxy compound $(-)-13(45 \% \text { yield, } \sim 99 \% \text { ee })^{8}$ and acetate $(+)-$ 14 (46\% yield, $\sim 99 \%$ ee) ${ }^{8}$ with high enantioselectivity and in preparatively useful yields (Scheme 2). ${ }^{7,8}$ Both $(-)-13$ and (+)-14 are serviceable for the synthesis of

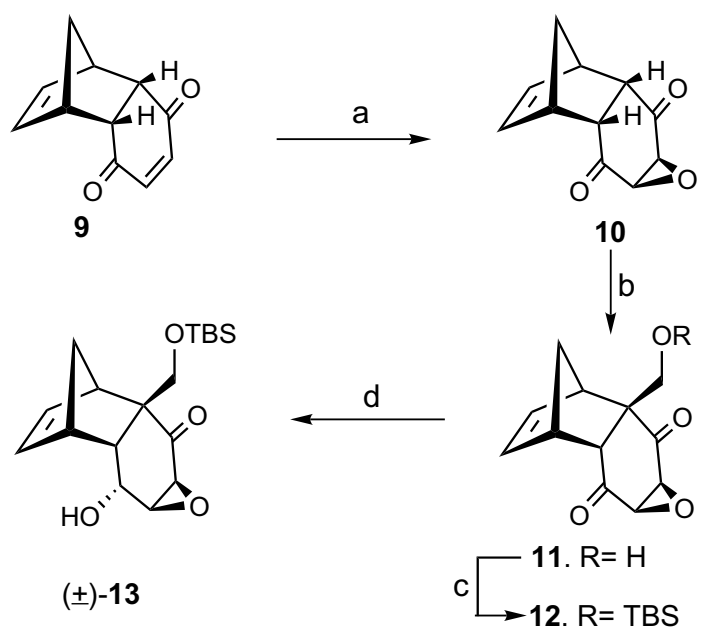

Scheme 1. Reagents and conditions: (a) $30 \% \mathrm{H}_{2} \mathrm{O}_{2}, 10 \% \mathrm{Na}_{2} \mathrm{CO}_{3}$, acetone, $0{ }^{\circ} \mathrm{C}, 96 \%$; (b) 0.1 equiv DBU, $40 \%$ formalin, THF, $0{ }^{\circ} \mathrm{C}, 95 \%$; (c) TBSCl, imid. DMAP, DMF, rt, $92 \%$; (d) $\mathrm{NaBH}_{4}, \mathrm{MeOH},-15^{\circ} \mathrm{C}$, $81 \%$.
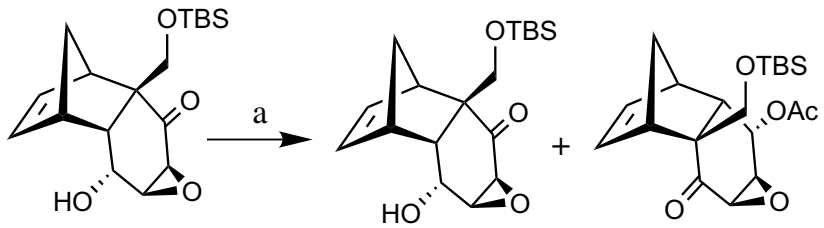

$( \pm)-13$

$(-)-13$

(+)-14

Scheme 2. Reagents and conditions: (a) Lipase PS-D (Amano), vinyl acetate, rt, 28 h. (-)-13, 45\%, (+)-14, 46\%.

diverse natural products and herein we describe a few syntheses emanating from (+)-14.

Enantiopure tricyclic acetate (+)-14 on thermal activation underwent facile retro-Diels-Alder reaction to eliminate cyclopentadiene and deliver epoxyquinone derivative (+)-15 (Scheme 3). ${ }^{7}$ Acetate hydrolysis in $(+)-15$ gave $(+)-16$ and further TBS deprotection furnished the natural product (+)-epiepoxydon $\mathbf{5 b}\left([\alpha]_{\mathrm{D}}\right.$ $+250, c$ 1.4, EtOH; lit. $\left.^{1 \mathrm{f}, 2 \mathrm{k}}[\alpha]_{\mathrm{D}}(+)-256, c 0.8, \mathrm{EtOH}\right)^{7}$ whose spectral data were found to be identical with those reported in the literature. ${ }^{1 \mathrm{f}, 2 \mathrm{k}}$

Hydroxy-enone (+)-16 was also suitable for the synthesis of the natural product (+)-epoxydon 5a and this required stereochemical inversion of the secondary hydroxyl group. Consequently, (+)-16 was directly subjected to the Mitsunobu protocol ${ }^{9}$ to deliver the hydroxyl inverted product $(+)-\mathbf{1 7}$ after hydrolysis (Scheme 4). ${ }^{7}$ TBS-deprotection in (+)-17 led to (+)-epoxydon 5a $\left([\alpha]_{\mathrm{D}}+98, c\right.$ 1.0, EtOH; lit. ${ }^{1 \mathrm{e}}[\alpha]_{\mathrm{D}}+102, c$ 1.0, EtOH $)$ and its spectral characteristics were found to be identical to those reported ${ }^{1 \mathrm{le}}$ for the natural product (Scheme 4). ${ }^{7}$

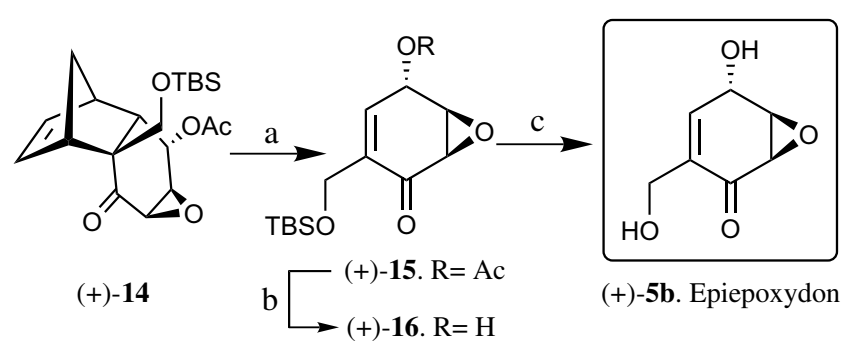

Scheme 3. Reagents and conditions: (a) diphenyl ether, $240^{\circ} \mathrm{C}, 5 \mathrm{~min}$, 93\%; (b) $\mathrm{LiOH}, \mathrm{MeOH}, 0{ }^{\circ} \mathrm{C}, 75 \%$; (c) $\mathrm{HF}$-pyridine, THF, $0{ }^{\circ} \mathrm{C}, 80 \%$. 


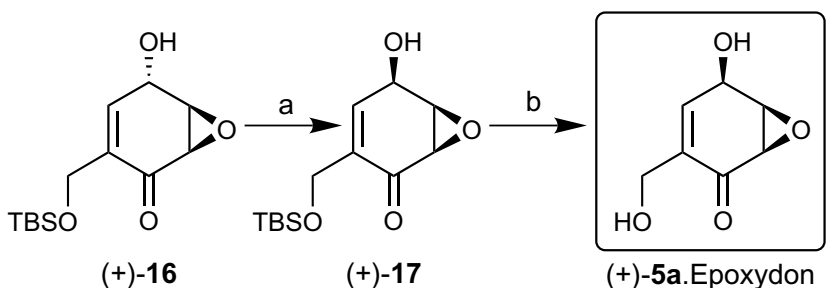

$(+)-16$
(+)-5a.Epoxydon

Scheme 4. Reagents and conditions: (a) (i) $\mathrm{PPh}_{3}$, DIAD, PNBA, THF, $-50{ }^{\circ} \mathrm{C}$ to $\mathrm{rt}$; (ii) $\mathrm{LiOH}, \mathrm{MeOH}, 0{ }^{\circ} \mathrm{C}, 65 \%$ (two steps). (b) $\mathrm{HF}-$ pyridine, THF, $0{ }^{\circ} \mathrm{C}, 76 \%$.

This synthesis, to our knowledge, is the first enantioselective synthesis of the natural product, $(+)$-epoxydon. ${ }^{10}$

For the synthesis of (-)-phyllostine, the hydroxyl group in (+)-16 was subjected to oxidation with PDC to give $(-)-18$ and further TBS-deprotection led to the epoxyquinone natural product $(-)-4\left([\alpha]_{\mathrm{D}}-108, c 1.61\right.$, EtOH; lit. ${ }^{1 \mathrm{~d}}[\alpha]_{\mathrm{D}}-105, c 1.0$, EtOH), Scheme $5 .{ }^{7}$ The spectral data for our synthetic (-)-phyllostine were found to be identical with those reported for the natural product.

Monocyclic acetate (+)-15 (Scheme 3) was considered as a suitable starting point for accessing the precursor 7 for a synthesis of (-)-panepophenanthrin $\mathbf{8}$, the antipode of the natural product. $^{3}$ It has been shown by others ${ }^{4 a, b}$ and $\mathrm{us}^{4 \mathrm{c}}$ that 7 undergoes spontaneous dimerization via a biomimetic Diels-Alder reaction to panepophenanthrin 8. Thus, accessing 7 became our penultimate objective. TBS deprotection in (+)-15 gave (+)-19 and further DIBAL- ${ }^{11}$ reduction of the carbonyl group proceeded under chelation control to furnish diol (+)20 as a single diastereomer (Scheme 6). ${ }^{7}$ The primary hydroxyl group in diol (+)-20 was chemoselectively oxidized in the TEMPO- $\mathrm{O}_{2}-\mathrm{CuCl}^{12}$ milieu to furnish aldehyde (+)-21. Horner-Wittig olefination in the hydroxyaldehyde 21 proceeded smoothly to render the $(E)$ - $\alpha, \beta$-unsaturated ester $(+)$-22 in good yield (Scheme 6). ${ }^{7}$ At this stage, it was necessary to carry out a methyl lithium addition to the ester carbonyl group of (+)-22 to deliver the desired side chain present in 7 . However, the presence of the acetate group in (+)-22 made this manoeuvre extremely messy and difficult to execute and therefore a more circuitous approach at the expense of a few additional steps was adopted. Acetate hydrolysis in (+)-22 was uneventful and led to the diol $(+)-23$ in which one hydroxyl group was regioselectively protected as its TBS-derivative (+)-24 (Scheme 7). ${ }^{7}$ Addition of

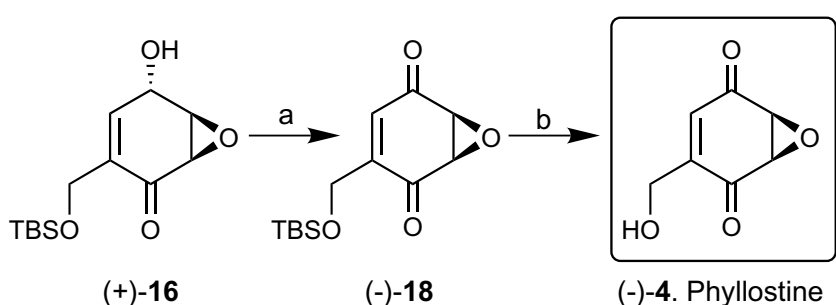

Scheme 5. Reagents and conditions: (a) PDC, DCM, $0{ }^{\circ} \mathrm{C}, 89 \%$; (b) $\mathrm{HF}$-pyridine, THF, $0{ }^{\circ} \mathrm{C}, 72 \%$.

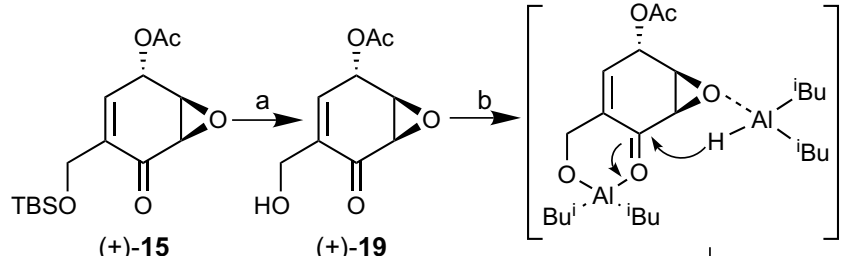

$(+)-15$

$(+)-19$<smiles>[3H]CC</smiles>

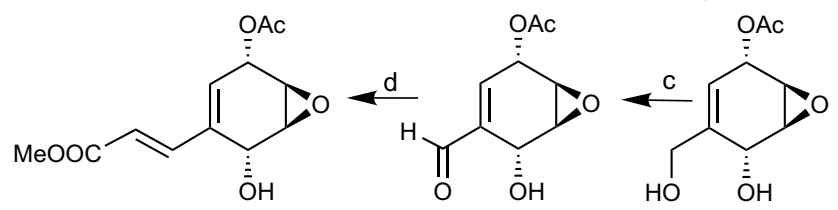

$(+)-22$

$(+)-21$

$(+)-20$

Scheme 6. Reagents and conditions: (a) $\mathrm{HF}-$ pyridine, THF, $0{ }^{\circ} \mathrm{C}, 92 \%$; (b) DIBAL-H, THF, $-78^{\circ} \mathrm{C}, 72 \%$; (c) TEMPO, $\mathrm{O}_{2}, \mathrm{CuCl}, \mathrm{DMF}, \mathrm{rt}$, $81 \%$; (d) $\mathrm{Ph}_{3} \mathrm{P}=\mathrm{CHCOOMe}$, benzene, rt, $94 \%$.

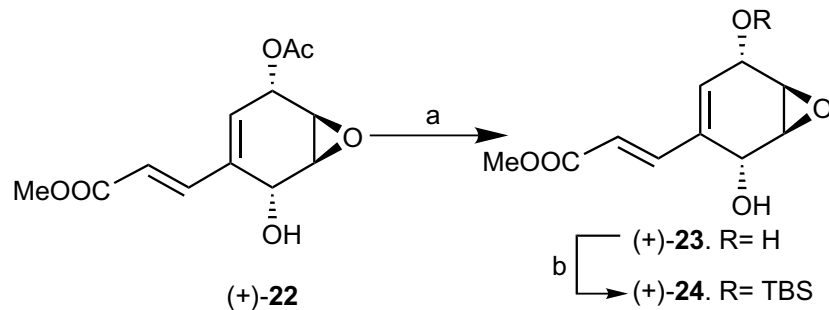

$(+)-22$

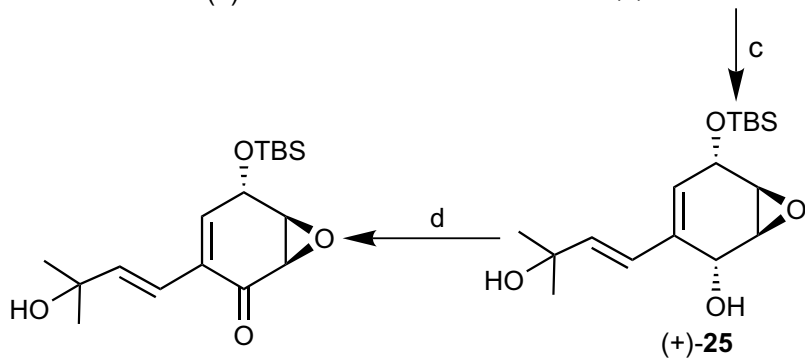

$(+)-26$

$(+)-25$

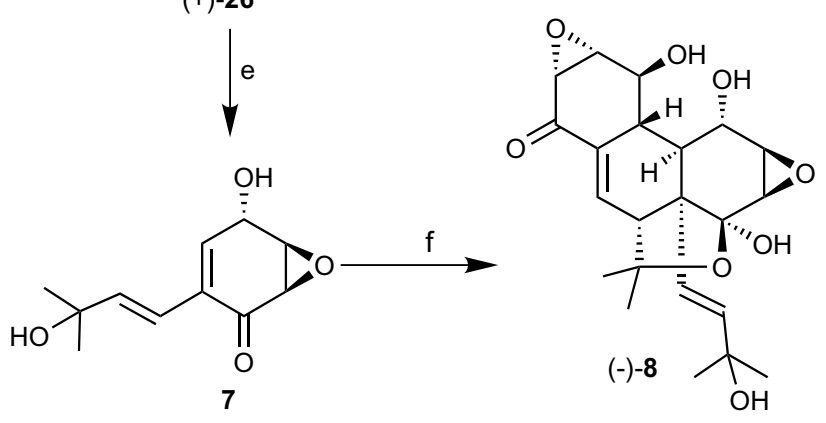

Scheme 7. Reagents and conditions: (a) $\mathrm{LiOH}, \mathrm{MeOH}, 0{ }^{\circ} \mathrm{C}, 88 \%$; (b) TBSOTf, imid. DMAP, DMF, rt, 71\%; (c) MeLi, THF, $0{ }^{\circ} \mathrm{C}, 60 \%$; (d) $\mathrm{MnO}_{2}, \mathrm{DCM}$, rt, $74 \%$; (e) $\mathrm{HF}$-pyridine, THF, $0^{\circ} \mathrm{C}, 94 \%$; (f) neat, $30 \mathrm{~h}$, $82 \%$.

methyllithium to $(+)-\mathbf{2 4}$ was now smooth and delivered $(+)-25$. Oxidation of the allylic hydroxyl group in (+)25 furnished the enone $(+)-\mathbf{2 6}^{7}$ and TBS deprotection led to the monomeric precursor 7 of the natural product panepophenanthrin (Scheme 7). When 7 was left neat under ambient conditions $\left(\sim 26^{\circ} \mathrm{C}\right)$ for $30 \mathrm{~h}$, it began 
to solidify and was transformed into a single dimeric product (-)-8 through a stereospecific intermolecular Diels-Alder reaction. ${ }^{13}$ The spectral data for $(-)-\mathbf{8}$ were identical with that of the natural product but had a rotation $\left([\alpha]_{\mathrm{D}}-147, c 1.0, \mathrm{MeOH}\right)$ opposite in sign to that of the natural product $\left(\right.$ lit. $\left.^{3}[\alpha]_{\mathrm{D}}+149.8, c 1.0, \mathrm{MeOH}\right){ }^{7}$ Thus, the first synthesis of the antipode of the biologically potent natural product panepophenanthrin has been achieved and its biological activity profile is being evaluated.

In short, we have devised a simple enzyme mediated strategy to access chiral building blocks for the synthesis of a range of biologically active epoxyquinone natural products from readily available starting materials. This versatile approach has resulted in the short syntheses of natural products $(-)$-phyllostine, $(+)$-epoxydon, $(+)$-epiepoxydon and (-)-panepophenanthrin.

\section{Acknowledgements}

K.I. thanks CSIR, India for the award of a research fellowship. This work was supported by the Chemical Biology Unit of JNCASR at the Indian Institute of Science, Bangalore. The lipase used in this study was a gift from Dr. Y. Hirose of Amano Pharmaceutical Co. Ltd., Japan.

\section{References and notes}

1. (+)-Epoformin: (a) Yamamoto, I.; Mizuta, E.; Henmi, T.; Yamano, T.; Yamatodani, S. J. Takeda Res. Lab. 1973, 32, 532; Scott, A. I.; Zamir, L.; Phillips, G. T.; Yalpani, M. Bioorg. Chem. 1973, 2, 124; (+)-epiepoformin: (b) Nagasawa, H.; Suzuki, A.; Tamura, S. Agr. Biol. Chem. 1978, 42, 1303; (-)-Theobroxide: (c) Nakamori, K.; Matsuura, H.; Yoshihara, T.; Ichihara, A.; Koda, Y. Phytochemistry 1994, 35, 835; Yoshihara, T.; Ohmori, F.; Nakamori, K.; Amanuma, M.; Tsutsumi, T.; Ichihara, A.; Matsuura, H. J. Plant Growth Reg. 2000, 19, 457; (-)phyllostine: (d) Sakamura, S.; Ito, J.; Sakai, R. Agr. Biol. Chem. 1970, 34, 153; Sakamura, S.; Ito, J.; Sakai, R. Agr. Biol. Chem. 1971, 35, 105; Kerr, K. A. Acta Crys. 1986, C42, 887; (+)-epoxidon: (e) Close, A.; Mauli, R.; Sigg, H. P. Helv. Chim. Acta 1966, 49, 204; Sakamura, S.; Niki, H.; Obata, Y.; Sakai, R.; Matsumoto, T. Agr. Biol. Chem. 1969, 33, 698; (+)-epiepoxidon: (f) Nagasawa, H.; Suzuki, A.; Tamura, S. Agr. Biol. Chem. 1978, 42, 1303; Sekiguchi, J.; Gaucher, G. M. Biochem. J. 1979, 182, 445; (+)harveynone: (g) Nagata, T.; Hirrota, A. Biosci. Biotechnol. Biochem. 1992, 56, 810.

2. For the total synthesis of (-)-phyllostine, (+)-epiepoxidon and related natural products, see: (a) Ichihara, A.; Oda, K.; Sakamura, S. Agr. Biol. Chem. 1971, 35, 445; (b) Ichihara, A.; Oda, K.; Sakamura, S. Tetrahedron Lett. 1972, 5105; (c) Ichihara, A.; Oda, K.; Sakamura, S. Agr. Biol. Chem. 1974, 38, 163; (d) Ichihara, A.; Kimura, R.; Oda, K.; Sakamura, S. Tetrahedron Lett. 1976, 4741; (e) Ichihara, A.; Moriyasu, K.; Sakamura, S. Agr. Biol. Chem. 1978, 42, 2421; (f) Teh-Wei Chou, D.; Ganem, B. J. Am. Chem. Soc. 1980, 102, 7987; (g) Ichihara, A.; Kimura, R.; Oda, K.; Moriyasu, K.; Sakamura, S. Agr. Biol. Chem. 1982, 46, 1879; (h) Ichihara, A. Synthesis 1987, 9, 207; (i) Kamikubu, T.; Ogasawara, K. Tetrahedron Lett. 1995, 36,
1685; (j) Graham, A. E.; McKerrecher, D.; Davies, D. H.; Taylor, R. J. K. Tetrahedron Lett. 1996, 37, 7445; (k) Kamikubu, T.; Hiroya, K.; Ogasawara, K. Tetrahedron Lett. 1996, 37, 499; (1) Liu, Z.; Li, H.; Chen, S. Huaxue Xuebao 1997, 55, 611; (m) Johnson, C. R.; Miller, M. W. J. Org. Chem. 1997, 62, 1582; (n) Yoshida, N.; Konno, H.; Kamikubu, T.; Takahashi, M.; Ogasawara, K. Tetrahedron: Asymmetry 1999, 10, 3849; (o) Barros, M. T.; Maycock, C. D.; Ventura, M. R. Tetrahedron 1999, 55, 3233; (p) Barros, M. T.; Maycock, C. D.; Ventura, M. R. Chem. Eur. J. 2000, 6, 3991; (q) Shimizu, H.; Okamura, H.; Yamashita, N.; Iwagawa, T.; Nakatani, M. Tetrahedron Lett. 2001, 42, 8649; (r) Genski, T.; Taylor, R. J. K. Tetrahedron Lett. 2002, 43, 3573; (s) Tachihara, T.; Kitahara, T. Tetrahedron 2003, 59, 1773; (t) Okamura, H.; Shimizu, H.; Yamashita, N.; Iwagawa, T.; Nakatani, M. Tetrahedron 2003, 59, 10159.

3. Sekizawa, R.; Ikeno, S.; Nakamura, H.; Naganawa, H.; Matsui, S.; Iinuma, H.; Takeuchi, T. J. Nat. Prod. 2002, 65,1491

4. (a) Lei, X.; Johnson, R. P.; Porco, J. A., Jr. Angew. Chem., Int. Ed. 2003, 42, 3913; (b) Moses, J. E.; Commeiras, L.; Baldwin, J. E.; Adlington, R. M. Org. Lett. 2003, 5, 2987; (c) Mehta, G.; Ramesh, S. S. Tetrahedron Lett. 2004, 45, 1985.

5. (a) Mehta, G.; Islam, K. Tetrahedron Lett. 2003, 44, 3569; (b) Mehta, G.; Islam, K. Org. Lett. 2004, 6, 807; (c) Mehta, G.; Pan, S. C. Org. Lett. 2004, 6, 811; (d) Mehta, G.; Islam, K. Tetrahedron Lett. 2004, 45, 3611; (e) Mehta, G.; Roy, S. Org. Lett. 2004, 6, 2389.

6. Cookson, R. C.; Crundwell, E.; Hill, R. R.; Hudec, J. J. Chem. Soc. 1964, 9, 3062

7. All new compounds were fully characterised on the basis of IR, ${ }^{1} \mathrm{H}$ NMR, ${ }^{13} \mathrm{C}$ NMR, mass data. Spectral data of selected compounds: (-)-13: $[\alpha]_{\mathrm{D}}^{24}:-19.1$ ( c 1.15, $\left.\mathrm{CHCl}_{3}\right)$; ${ }^{1} \mathrm{H}$ NMR $\left(300 \mathrm{MHz}, \mathrm{CDCl}_{3}\right): \delta 6.22(\mathrm{~s}, 2 \mathrm{H}), 4.62(\mathrm{dt}, 1 \mathrm{H}$, $J=2.7,9.9 \mathrm{~Hz}), 4.42(\mathrm{~d}, 1 \mathrm{H}, J=9.9 \mathrm{~Hz}), 3.57(\mathrm{~d}, 1 \mathrm{H}$, $J=9.9 \mathrm{~Hz}), 3.52(\mathrm{dd}, 1 \mathrm{H}, J=3,3.9 \mathrm{~Hz}), 3.26(\mathrm{~d}, 1 \mathrm{H}$, $J=3.9 \mathrm{~Hz}), 3.17(\mathrm{~s}, 1 \mathrm{H}), 2.92(\mathrm{~s}, 1 \mathrm{H}), 2.32(\mathrm{dd}, 1 \mathrm{H}$, $J=3.3,7.2), 1.44(\mathrm{~d}, 1 \mathrm{H}, \quad J=9.3 \mathrm{~Hz}), 1.37(\mathrm{~d}, 1 \mathrm{H}$, $J=9.3 \mathrm{~Hz}), \quad 0.87 \quad(\mathrm{~s}, \quad 9 \mathrm{H}), \quad 0.02 \quad(\mathrm{~s}, \quad 6 \mathrm{H}) ;{ }^{13} \mathrm{C} \quad \mathrm{NMR}$ $\left(75 \mathrm{MHz}, \mathrm{CDCl}_{3}\right): \delta 206.5,136.9,136.5,69.4,66.9,62.7$, 59.9, 54.6, 49.1, 46.1, 45.9, 44.9, 25.8 (3C), 18.2, -5.5, -5.6 ; HRMS (ES) $\mathrm{m} / \mathrm{z}$ calcd for $\mathrm{C}_{18} \mathrm{H}_{27} \mathrm{O}_{4} \mathrm{SiK}[\mathrm{M}+\mathrm{K}]^{+}$: 375.1394, found: 375.1400 . (+)-14: $[\alpha]_{\mathrm{D}}^{24}+24\left(c 1.95, \mathrm{CHCl}_{3}\right)$; ${ }^{1} \mathrm{H}$ NMR $\left(300 \mathrm{MHz}, \mathrm{CDCl}_{3}\right): \delta 6.16-6.13(\mathrm{~m}, 1 \mathrm{H})$, 5.94-5.91 (m, 1H), $5.80(\mathrm{dd}, 1 \mathrm{H}, J=3.0,7.5), 4.32(\mathrm{~d}$, $1 \mathrm{H}, J=9.9 \mathrm{~Hz}), 3.60(\mathrm{~d}, 1 \mathrm{H}, J=9.6 \mathrm{~Hz}), 3.40(\mathrm{dd}, 1 \mathrm{H}$, $J=2.7,3.9 \mathrm{~Hz}), 3.26(\mathrm{~d}, 1 \mathrm{H}, J=3.6 \mathrm{~Hz}), 3.16(\mathrm{~s}, 1 \mathrm{H}), 2.79$ $(\mathrm{s}, 1 \mathrm{H}), 2.43(\mathrm{dd}, 1 \mathrm{H}, J=3.3,7.8 \mathrm{~Hz}), 2.10(\mathrm{~s}, 3 \mathrm{H}), 1.42(\mathrm{~d}$, $1 \mathrm{H}, J=9.3 \mathrm{~Hz}), 1.34(\mathrm{~d}, 1 \mathrm{H}, J=9.3 \mathrm{~Hz}), 0.88(\mathrm{~s}, 9 \mathrm{H}), 0.03$ $(\mathrm{s}, 6 \mathrm{H}) ;{ }^{13} \mathrm{C} \mathrm{NMR}\left(75 \mathrm{MHz}, \mathrm{CDCl}_{3}\right): \delta 205.3,169.9,136.1$, 135.9, 69.3, 66.7, 61.7, 57.2, 54.4, 46.9, 46.6, 45.7, 44.9, 25.8 (3C), 21.26, 18.2, -5.5, -5.6; HRMS (ES) $\mathrm{m} / \mathrm{z}$ calcd for $\mathrm{C}_{20} \mathrm{H}_{30} \mathrm{O}_{5} \mathrm{SiK}[\mathrm{M}+\mathrm{K}]^{+}:$: 417.1500, found: 417.1492. (+)5b: $[\alpha]_{\mathrm{D}}^{25}+250$ (c 1.40, EtOH); ${ }^{1} \mathrm{H}$ NMR $(300 \mathrm{MHz}$, $\left.\mathrm{CD}_{3} \mathrm{COCD}_{3}\right): \delta$ 6.72-6.69 $(\mathrm{m}, 1 \mathrm{H}), 4.92(\mathrm{~d}, 1 \mathrm{H}$, $J=7.5 \mathrm{~Hz}), 4.66-4.63(\mathrm{~m}, 1 \mathrm{H}), 4.30-4.10(\mathrm{~m}, 3 \mathrm{H}), 3.78-$ $3.76(\mathrm{~m}, 1 \mathrm{H}), 3.40(\mathrm{~d}, 1 \mathrm{H}, J=3.6 \mathrm{~Hz}) ;{ }^{13} \mathrm{C} \mathrm{NMR}(75 \mathrm{MHz}$, $\left.\mathrm{CD}_{3} \mathrm{COCD}_{3}\right): \delta 194.35,139.30,137.07,63.25,59.0,58.79$, 54.1; HRMS (ES) $\mathrm{m} / z$ calcd for $\mathrm{C}_{7} \mathrm{H}_{8} \mathrm{O}_{4} \mathrm{Na}[\mathrm{M}+\mathrm{Na}]^{+}$: 179.0320, found: 179.0314. (+)-5a: $[\alpha]_{\mathrm{D}}^{25}+98.0(c$ 1.0, EtOH); ${ }^{1} \mathrm{H} \quad \mathrm{NMR} \quad\left(300 \mathrm{MHz}, \quad \mathrm{CD}_{3} \mathrm{COCD}_{3}\right): \delta 6.50(\mathrm{~d}, \quad 1 \mathrm{H}$, $J=1.8 \mathrm{~Hz}), 4.91(\mathrm{~d}, 1 \mathrm{H}, J=7.5 \mathrm{~Hz}), 4.80-4.77(\mathrm{~m}, 1 \mathrm{H})$, $4.24-4.06(\mathrm{~m}, 3 \mathrm{H}), 3.80(\mathrm{~d}, 1 \mathrm{H}, J=3.0,6.6 \mathrm{~Hz}), 3.34$ $(\mathrm{d}, 1 \mathrm{H}, J=4.2 \mathrm{~Hz}) ;{ }^{13} \mathrm{C} \mathrm{NMR}\left(75 \mathrm{MHz}, \mathrm{CD}_{3} \mathrm{COCD}_{3}\right): \delta$ 194.5, 141.4, 135.2, 65.5, 59.1, 55.0, 54.0; HRMS (ES) $\mathrm{m} / \mathrm{z}$ calcd for $\mathrm{C}_{7} \mathrm{H}_{8} \mathrm{O}_{4} \mathrm{Na}[\mathrm{M}+\mathrm{Na}]^{+}$: 179.0320 , found: 179.0310 . $(-)-4:[\alpha]_{\mathrm{D}}^{24}:-108\left(c\right.$ 1.61, EtOH); ${ }^{1} \mathrm{H}$ NMR $(300 \mathrm{MHz}$, 
$\left.\mathrm{CDCl}_{3}\right): \delta 6.67(\mathrm{dd}, 1 \mathrm{H}, J=1.9,3.8 \mathrm{~Hz}), 4.56(\mathrm{~d}, 1 \mathrm{H}$, $J=17.4 \mathrm{~Hz}), 4.38(\mathrm{~d}, 1 \mathrm{H}, 17.4 \mathrm{~Hz}), 3.84-3.81(\mathrm{~m}, 2 \mathrm{H}), 2.25$ (br s, $1 \mathrm{H}) ;{ }^{13} \mathrm{C} \mathrm{NMR}\left(75 \mathrm{MHz}, \mathrm{CDCl}_{3}\right): \delta 192.0,191.3$, 148.1, 131.0, 59.2, 54.0 (2C); HRMS (ES) $\mathrm{m} / \mathrm{z}$ calcd for $\mathrm{C}_{7} \mathrm{H}_{6} \mathrm{O}_{4} \mathrm{~K}[\mathrm{M}+\mathrm{K}]^{+}:$192.9903, found: $102.9900 .(-)-8:[\alpha]_{\mathrm{D}}^{24}$ : -147.0 (c 1.0, MeOH); ${ }^{1} \mathrm{H}$ NMR $\left(300 \mathrm{MHz}, \mathrm{CD}_{3} \mathrm{OD}\right): \delta$ $6.81(\mathrm{dd}, J=5.0,3.0 \mathrm{~Hz}, 1 \mathrm{H}), 5.99(\mathrm{~d}, J=16.2 \mathrm{~Hz}, 1 \mathrm{H})$, $5.68(\mathrm{~d}, J=16.2 \mathrm{~Hz}, 1 \mathrm{H}), 4.55$ (br s, $1 \mathrm{H}), 4.35$ (br s, $1 \mathrm{H})$, $3.84(\mathrm{t}, J=3.4 \mathrm{~Hz}, 1 \mathrm{H}), 3.50(\mathrm{t}, J=3.2 \mathrm{~Hz}, 1 \mathrm{H}), 3.42(\mathrm{~d}$, $J=4.0 \mathrm{~Hz}, 1 \mathrm{H}), 3.35(\mathrm{dd}, J=5.0,1.6 \mathrm{~Hz}, 1 \mathrm{H}), 3.31(\mathrm{~d}$, $J=4 \mathrm{~Hz}, 1 \mathrm{H}), 2.32($ br d, $J=10.0 \mathrm{~Hz}, 1 \mathrm{H}), 2.03(\mathrm{br} \mathrm{d}$, $J=9.7 \mathrm{~Hz}, 1 \mathrm{H}), 1.45(\mathrm{~s}, 3 \mathrm{H}), 1.35(\mathrm{~s}, 3 \mathrm{H}), 1.20(\mathrm{~s}, 3 \mathrm{H})$, $1.17(\mathrm{~s}, 3 \mathrm{H}) .{ }^{13} \mathrm{C} \mathrm{NMR}\left(75 \mathrm{MHz}, \mathrm{CD}_{3} \mathrm{OD}\right): \delta 196.3,143.0$, 139.9, 138.8, 129.3, 102.7, 79.2, 71.8, 69.0, 66.2, 60.7, 57.4, $57.2,57.1,55.6,55.1,51.2,50.0,32.3,30.3,29.5,26.2$; HRMS (ES) $m / z$ calcd for $\mathrm{C}_{22} \mathrm{H}_{28} \mathrm{O}_{8} \mathrm{Na} \quad[\mathrm{M}+\mathrm{Na}]^{+}$: 443.1682, found: 443.1698 .

8. The enantiomeric excess (ee) was determined through ${ }^{1} \mathrm{H}$ NMR analyses based on the integration of the acetate methyl groups after the addition of chiral shift reagent tris[3-(trifluoromethylhydroxymethylene)-(+)-camphorato] europium (III). Procedure for enzymatic kinetic resolution: A mixture of racemic epoxy-alcohol 13 (1 g, $2.97 \mathrm{mmol})$, vinyl acetate $(25 \mathrm{~mL})$ and Amano lipase
PS-D immobilized on celite $(1 \mathrm{~g})$ was stirred for $28 \mathrm{~h}$ at room temperature. The reaction mixture was monitored and after $\sim 50 \%$ conversion it was filtered through a pad of celite and the filtrate was concentrated. The crude product was subjected to column chromatography on silica gel and eluted first with $10 \%$ ethyl acetate in hexane to furnish $516 \mathrm{mg}(46 \%)$ of keto-acetate $(+)-14\left([\alpha]_{\mathrm{D}}+24, c 1.95\right.$ $\mathrm{CHCl}_{3}, \sim 99 \%$ ee). Further elution with $25 \%$ ethyl acetate in hexane gave $450 \mathrm{mg}(45 \%)$ of $(-)-\mathbf{1 3}\left([\alpha]_{\mathrm{D}}-19.1, c 1.15\right.$, $\mathrm{CHCl}_{3}, \sim 99 \%$ ee).

9. Martin, S. F.; Dodge, J. A. Tetrahedron Lett. 1991, 32, 4741.

10. Absolute configuration of (+)-epoxydon was determined by $\mathrm{CD}$ studies ${ }^{\mathrm{le}}$ and further confirmed by conversion to $(-)$-phyllostine. ${ }^{1 \mathrm{~d}}$

11. Kiyooka, S.; Kuroda, H.; Shimasaki, Y. Tetrahedron Lett. 1986, 27, 3009.

12. Semmelhack, M. F.; Schmid, C. R.; Cortes, D. A.; Chou, C. S. J. Am. Chem. Soc. 1984, 106, 3374.

13. The stereoselectivity in the Diels-Alder dimerisation of $\mathbf{7}$, leading to the natural product $(+)-8$ is quite remarkable and several explanations for it have been offered ${ }^{4 a, b}$ and elegantly probed both experimentally and computationally by the group of Porco. ${ }^{4 a}$ 